\title{
Programa Jóvenes en Acción en la Universidad de la Amazonia: una lectura desde los actores en cuestión
}

\author{
Carlos Alberto Gómez-Cano*, Verenice Sánchez-Castillo**, Yamil Hernando Rivera-Cortés***
}

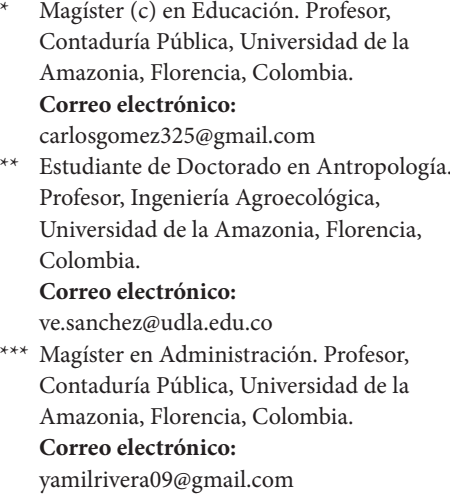

Recibido: 22 de septiembre del 2015 Aceptado: 9 de noviembre del 2015

Cómo citar este artículo: Gómez-Cano, C. A., Sánchez-Castillo, V. y Rivera-Cortés, Y.H. (2016). Programa Jóvenes en Acción en la Universidad de la Amazonia: una lectura desde los actores en cuestión. Cooperativismo y Desarrollo, 24(108), 157-173. doi: http://dx.doi.org/10.16925/ co.v24i108.1266

\section{Resumen}

Introducción: Jóvenes en Acción (PJEA) es un programa del gobierno nacional colombiano que busca apoyar los procesos de educación superior a través de transferencias monetarias condicionadas a jóvenes que estén cursando una tecnología o una formación profesional. En la Universidad de la Amazonia, este programa comenzó a funcionar desde el primer semestre del 2014 y actualmente beneficia a 2223 estudiantes del alma mater. Metodología: las herramientas empleadas consistieron en arqueo de archivo, revisión de bases de datos del programa, identificación de actores clave, realización de entrevistas y procesamiento de los datos en el software Altas Ti. Resultados: se evidenció un incremento periódico a través de cada semestre en cuanto a los beneficiarios y una disminución en los estudiantes inactivos y condicionados. Los imaginarios del programa por parte de los estudiantes son de éxito, pero a corto plazo y de impacto inmediato, lejos de lo que busca el programa mismo. Conclusiones: es preciso avanzar en dos líneas de interés que preocupan al programa, que son: la cobertura y el nivel de exigencia, los cuales se deben tener para sostenerse en este.

Palabras clave: educación, ejecutores, estudiante, imaginario. 


\title{
The Program Jóvenes en Acción at the Universidad de la Amazonía: A Reading from the Actors Concerned
}

\begin{abstract}
Introduction: Jóvenes en Acción (PJEA, after its Spanish initials) is a program of the national government of Colombia that aims to support higher education processes through conditional cash transfers to young people who are pursuing undergraduate studies. At the Universidad de la Amazonia, this program became operational from the first half of 2014 and currently benefits 2223 students at this alma mater. Methodology: The tools used were file checking, review of program databases, identification of key stakeholders, interviews, and data processing in the Altas Ti software. Results: A regular increase throughout each half regarding beneficiaries and a decrease in inactive and conditioned students were evidenced. The students' collective imaginary about the program is that it is successful but in the short term and has immediate impact, far from what the program itself is looking for. Conclusions: It is necessary to progress on two lines of interest that causes the program's concern, namely, coverage and level of demand, which should be present to support it.
\end{abstract}

Keywords: education, executors, student, imaginary.

\section{"Programa Jóvenes en Acción" na Universidad de la Amazonia: uma leitura a partir dos atores em questão}

\section{Resumo}

Introdução: Jóvenes en Acción (PJEA) é um programa do governo nacional colombiano que procura apoiar os processos de educação superior por meio de transferências monetárias condicionadas a jovens que estejam num curso tecnológico ou de formação profissional. Na Universidad de la Amazonia, esse programa começou a funcionar no primeiro semestre de 2014 e, atualmente, beneficia a 2223 estudantes do alma mater. Metodologia: as ferramentas empregadas neste estudo consistiram em busca em arquivo, revisão de bases de dados do programa, identificação de atores-chave, realização de entrevistas e processamento dos dados no software Altas Ti. Resultados: evidenciou-se um aumento periódico a cada semestre dos beneficiários e uma diminuição nos estudantes inativos e condicionados. Os imaginários do programa por parte dos estudantes são um sucesso, mas de curto prazo e de impacto imediato, longe do que o programa em si objetiva. Conclusões: é preciso avançar em duas linhas de interesse que preocupam o programa: a cobertura e o nível de exigência, que devem ter para se sustentar neste.

Palavras-chave: educação, executores, estudante, imaginário. 


\section{Introducción}

Jóvenes en Acción (JEA) es un programa del Gobierno nacional que busca fortalecer los procesos de formación para el trabajo de los jóvenes en condición de pobreza y vulnerabilidad, a través de un modelo de transferencias monetarias condicionadas (TMC). Su coordinación está a cargo de la Dirección de Ingreso Social (DIS) del Departamento para la Prosperidad Social (DPS), quien es la encargada de aplicar las políticas, planes, programas, estrategias y proyectos de transferencias monetarias dirigidas a reducir la vulnerabilidad de la población pobre; además de diseñar los instrumentos de verificación de compromisos de los beneficiarios de dichas transferencias monetarias de acuerdo con la política del Gobierno nacional definida para tal fin (DPS, 2013).

Los objetivos específicos del programa están relacionados con incentivar la demanda por educación de los jóvenes en acción en condición de pobreza y vulnerabilidad para acceder a la formación profesional, contribuir a la formación de sus capacidades y competencias para el trabajo, reducir el porcentaje de inactividad de ellos, generar movilidad social, brindar asesoría y acompañamiento en el desarrollo de su plan de mejoramiento de capacidades para la vida y promover la articulación de oferta institucional pública y privada en función de su demanda en la educación (dps, 2013).

El programa JEA implementa las estrategias para el logro de sus objetivos a través de dos componentes: formación y habilidades para la vida. El primero se refiere a aquellas actividades de carácter académico (teórico o práctico) que conforman el proceso de formación del joven participante y en el segundo aborda el fortalecimiento de aquellas aptitudes necesarias que permiten y facilitan la inserción del joven en el ámbito social y laboral, y que le contribuyen enfrentarse a los retos y desafíos de la vida diaria (DPS, 2013).

El modelo de gestión del Programa Jóvenes en Acción es manejado desde DIs y también se cuenta con profesionales/enlaces en las direcciones regionales donde opera el programa. Los enlaces son los encargados de la ejecución de los diferentes procesos operativos y de la verificación y seguimiento al cumplimiento de las responsabilidades de los diferentes actores locales e institucionales, involucrados con el programa en el territorio (DPS, 2013).
Jóvenes en Acción se articula con el rediseño del programa Más Familias en Acción ${ }^{1}$ con el fin de dar continuidad al proceso de acumulación de capital humano en la población que se graduó de este programa. De igual forma, el contexto institucional del programa es apoyado por el Departamento Nacional de Planeación (DNP), el Ministerio de Trabajo, el Ministerio de Educación Nacional (MEN), el Servicio Nacional de Aprendizaje (sENA), el Ministerio de la Hacienda y Crédito Público, la Agencia Nacional para la Superación de la Pobreza Extrema (ANSPE), la Unidad para la Atención y Reparación Integral a las Víctimas, el Instituto Colombiano de Bienestar Familiar (ICBF), las instituciones financieras, el programa Colombia Joven, la Mesa de Pobreza y la Mesa de Generación de Ingresos.

En el ámbito local, guarda estrecha relación con las autoridades locales (AL), las instituciones educativas (IE), las direcciones regionales del DPS, los centros de formación del SENA, las oficinas regionales del Servicio Público de Empleo (SPE) del SENA y los Profesional/Enlace Territorial del DPS (DPS, 2013).

El ciclo operativo está conformado por las siguientes actividades: focalización, registro, inscripción, priorización de la población objetivo, taller de participantes, proceso de registro inscripción y matrícula en la institución educativa, enrolamiento financiero, depuración de información, suspensión preventiva de giros, verificación de compromisos de los jóvenes, casos especiales de suspensión del giro, liquidación, entrega de incentivos, dispersión de incentivos, reintegros, pago de comisiones, novedades y gestión de quejas y reclamos (DPS, 2013).

Para garantizar la operatividad y correcto funcionamiento del programa, la DIs cuenta con un Comité de Coordinación y un Comité Operativo, que son las instancias que sirven a la articulación, coordinación, comunicación y aprobación de las decisiones de los programas a cargo de la Dirección. Además, se cuenta con el Comité Técnico Interinstitucional, conformado entre el SENA y el DPS, cuya función está relacionada con elaborar cronogramas de actividades, hacer seguimiento a la ejecución del convenio, evaluar los resultados obtenidos en las diferentes

\footnotetext{
1 Más Familias en Acción es un programa del Dps que ofrece a todas aquellas familias con niños, niñas y adolescentes menores de 18 años un apoyo económico con el fin de lograr una alimentación saludable, un crecimiento y desarrollo adecuado al tiempo que permanecen en un sistema escolar. Este programa apoya a las familias a través de incentivos económicos para salud y educación (DPS, 2013).
} 
actividades y garantizar la adecuada cooperación y coordinación entre las partes (DPS, 2013).

Además de lo anterior, en el marco del Comité Técnico del Convenio Interadministrativo SENA-DPS N. ${ }^{\circ}$ 053 de 2013, se acordó la creación del Comité de Casos Especiales, encargado de revisar los casos especiales de jóvenes que por inconsistencias en el proceso de verificación de compromisos, se les haya suspendido el giro de la transferencia monetaria condicionada (TMC). El comité define el proceso que debe seguirse para cada caso y el tiempo de respuesta que se tiene para dar respuesta al joven. Cabe mencionar que este está conformado por las instituciones educativas participantes en el programa y el DPS (DPS, 2013).

Los jóvenes elegibles del programa jea son colombianos entre 16 y 24 años de edad, que no se encuentren en condición de pérdida de sus derechos civiles, y que adicionalmente cumplan con por lo menos uno de los siguientes criterios de elegibilidad: 1) estar en el Sisben III, con uno de los puntajes establecidos dentro de las áreas geofiguras para la focalización poblacional, o 2) estar registrado en la Red Unidos; o 3) estar registrado en el RUV en condición de desplazamiento incluido; o 4) estar registrado en las listas censales de jóvenes indígenas; o 5) estar registrado en las listas censales para jóvenes con medida de protección del ICBF (DPS, 2013).

El joven en acción permanece en el programa el tiempo que dure su proceso de formación profesional, siempre y cuando mantenga el mínimo promedio exigido, según cada institución educativa donde el joven se encuentre en formación.

El registro es el paso previo a la inscripción del joven al Programa Jóvenes en Acción. Se encuentra a cargo del DPS y consiste en la actualización de los datos personales, de contacto y de nivel de formación del joven potencial participante interesado en el programa. La actualización de datos se realiza anualmente a partir de la verificación de la documentación exigida: documento de identidad en original y fotocopia legible y acta o diploma de grado en original o fotocopia. Dichos documentos son entregados por los medios que el DPs establece de manera previa a la convocatoria. (DPS, 2013).

La inscripción es el proceso mediante el cual el joven elegible seleccionado en el proceso de registro formaliza su intención de ingreso al programa. Un joven registrado que sea un Joven en Acción, participará en las siguientes actividades: 1) taller de potenciales participantes, 2) convocatoria de registro, inscripción y matrícula en un programa de formación profesional a cargo de la institución educativa universitaria o de la institución de formación para el trabajo y el desarrollo humano, y 3) jornadas de enrolamiento financiero. Producto de estas actividades, el joven potencial participante debe haberse matriculado en un programa de formación profesional en la institución educativa con la cual el DPs haya suscrito acuerdos/ convenios interinstitucionales (DPS, 2013).

Durante el proceso de inscripción, el DPs gestiona ante las instituciones educativas acuerdos particulares de acceso preferencial o diferencial de la población objetivo del programa y define la emisión de los reportes de avance del proceso de registro, inscripción y matrícula, a partir del listado o de la base de datos (BD) de jóvenes potenciales participantes del programa, que haya sido entregada por el DPS (DPS, 2013)

Seguido del proceso de inscripción, se procede a la depuración de la base de datos, con el fin de identificar inconsistencias o indicios de falsedad, según Resolución N. ${ }^{\circ} 2019$ del 6 de diciembre de 2012. Como producto de esta actividad, se generan acciones administrativas que permiten corregir la información. Si alguno de los jóvenes presenta una inconsistencia en las base de datos se procede a la suspensión preventiva del giro y se le comunicará para que realice los procesos respectivos para el protocolo de levantamiento de la suspensión preventiva. Este proceso está a cargo de los profesionales territoriales del DPS y de las instituciones educativas que después de la valoración de los elementos de conocimiento podrá: levantar la medida preventiva adoptada y reliquidar el valor de los incentivos a que haya tenido derecho el participante o excluir definitivamente al JEA de la base de datos (DPS, 2013).

La verificación de compromisos es el acto que se realiza mes a mes mediante el cual la institución educativa (SENA, universidades o instituciones de formación para el trabajo y el desarrollo humano) certifica al DPs que el joven participante de Jóvenes en Acción cumple con su proceso de formación de acuerdo con los parámetros establecidos por el programa y acordados con la institución educativa. Si en dicho proceso el DPs detecta alguna inconsistencia se le suspenderá el giro de la Transferencia Monetaria Condicionada, hasta que se aclaren las circunstancias que motivan la inconsistencia. Una vez se indaguen las causas, el DPS autoriza o cancela el giro, según las directrices que determine el Comité de Casos Especiales (DPS, 2013). 
Cumplido el proceso de verificación de compromisos, se procede a la dispersión de los incentivos, proceso mediante el cual la entidad bancaria realiza las órdenes de pago de los giros y los abonos a las cuentas de los jóvenes participantes del programa. Una vez recibido el detalle de los incentivos que le corresponden a cada participante, como resultado de la liquidación realizada por el programa, se ejecutan las siguientes actividades: 1) solicitud de recursos a la Subdirección Financiera del DPS y ordenación del pago por parte del Ordenador del Gasto, 2) validación de archivos, 3) elaboración de cartas de autorización débito para que los bancos realicen la dispersión de los recursos a la cuenta del joven, 4) solicitud mediante memorando del reproceso de los pagos rechazados. Se repiten los puntos 2 y 3 (DPS, 2013).

Si el joven beneficiario del programa JEA presenta alguna inconformidad, podrá expresarla a las direcciones regionales del DPS, a través de los canales de atención al ciudadano establecidos por la entidad, y se enviará copia al nivel nacional para el seguimiento correspondiente, en las categorías de peticiones, quejas y reclamos (DPs, 2013).

Los compromisos del Joven en Acción con el programa implican que el joven debe asistir a su proceso de formación de manera regular en la institución de educación superior o en la institución de formación para el trabajo y el desarrollo humano correspondiente (por ejemplo, el SENA). Adicionalmente, debe cumplir con las obligaciones/deberes/compromisos establecidos en el reglamento académico/estudiantil de la institución. De igual forma, debe responder por lo exigido en el componente de Habilidades para la vida; en este sentido, deberá asistir regularmente a los talleres y demás actividades que el componente programa (DPs, 2013).

La frecuencia de verificación es mensual a partir del inicio de clases de cada institución educativa. Las condiciones de cada institución se describen en el acuerdo interinstitucional que el DPs establezca con cada institución educativa (DPS, 2013).

Cada joven recibe un estímulo monetario, es decir, la transferencia monetaria condicionada (TMC) que corresponde al monto girado directamente al joven participante, durante todo el proceso de formación del JEA, previa verificación del cumplimiento de compromisos; por valor de 200000 cada mes (DPS, 2013).

El joven sale del programa cuando haya culminado con éxito su proceso de formación profesional y se haya certificado/graduado en el tiempo máximo establecido por el programa o por otras razones que ameriten su salida temprana. También existe la posibilidad de reingreso (DPS, 2013).

A nivel regional, se tiene que el pJEA llegó a la Universidad de la Amazonia a través de las Transferencias Monetarias Condicionadas en el primer semestre del 2014, ha beneficiado a más de 2200 jóvenes; sin embargo, se desconoce el impacto real que este programa ha tenido sobre los jóvenes, el cual va mucho más allá de si recibieron o no el apoyo económico. Si bien el interés del programa es precisamente mejorar la calidad académica, liberar tiempo para estudiar, conviene entonces revisar si efectivamente esto se está logrando, de igual forma, poder conocer si los jóvenes beneficiados pudieron dejar de trabajar y dedicar más tiempo a sus estudios, y cuál es la percepción acerca del programa que tienen los directamente implicados.

\section{El PJEA en la Universidad de la Amazonia}

\section{Caracterización del Programa Jóvenes en Acción en la Universidad de la Amazonia}

\section{Historia del programa en la Universidad de la Amazonia}

El programa JEA llegó a la Universidad de la Amazonia, finalizando el 2012; la firma del convenio con el Departamento para la Prosperidad Social (DPS) tardó todo el 2013, razón por la cual la socialización y la invitación para ingresar a este, se inició a partir de enero del 2014.

Con el inicio del semestre, se empezó hacer la promoción del convenio, de los beneficios que podía traer para los estudiantes. Los encargados de esta labor fueron los funcionarios de la Oficina Administrativa de Relaciones Interinstitucionales (OARI) de la Universidad de la Amazonia, inicialmente solo una persona de la OARI y otra del DPS, se encargaron del manejo de la plataforma para el ingreso de las solicitudes, pero prontamente el tema se congestionó y surgió la necesidad de realizar capacitaciones a los estudiantes para que ellos mismos realizaran su ingreso. Así las cosas, el programa se promocionó a través del sitio web de la Universidad y por los salones a través de la estrategia de comunicación de voz a voz. La receptividad fue total, el Gobierno nacional tenía presupuestado para la Universidad de la Amazonia, un cupo para 
el programa de 800 estudiantes y a corte del semestre 2015-i, se están beneficiando más de 2200 (Sánchez, comunicación personal, 10 de septiembre del 2015).

\section{Los requisitos}

El ingreso de un estudiante por primera vez al PJEA está determinado por la entrega de toda la documentación y la matricula como mínimo del $78 \%$ de los créditos que tiene en ese semestre. La selección del estudiante depende exclusivamente del DPS y su sistema de información, la plataforma sija está cruzada con el Departamento Nacional de Planeación, con información como cédula y Sisben, de esta manera, el sistema permite identificar si el joven está en otras bases de datos, como por ejemplo Famac, por lo cual es rechazado inmediatamente. En este orden de ideas, el sistema de selección, está programado de acuerdo con los censos que maneja el dnp y las bases de datos de otras instituciones de beneficios sociales del orden nacional que son cruzadas continuamente, emitiendo el juicio de acepto o no aceptado.

Existen unos requisitos generales de ingreso al PJEA que fueron expuestos anteriormente, los cuales son iguales a nivel nacional; no obstante, cuando el programa llegó a la Uniamazonia, no se tenía un nivel de promedio de calificaciones exigido para el ingreso. Es así como la Universidad y el Dps establecieron que este fuera 3,0; teniendo en cuenta que el rendimiento académico está condicionado por diferentes situaciones, como las necesidades básicas insatisfechas, la mala alimentación, el no acceso a una vida digna y el no tener una estabilidad emocional definida, son factores que hacen que su rendimiento sea bajo. Aunado a lo anterior, algunos son desplazados, están en condición de protección del ICBF, o se hallan en categoría de vulnerabilidad alta.

\section{De las suspensiones temporales}

Se entiende por suspensión temporal aquella TMC cuya entrega es aplazada temporalmente, mientras se subsana la causa de la suspensión. La causa más frecuente de la suspensión temporal en caso de la ua consiste en que los jóvenes ingresan al programa teniendo como documento de identificación la tarjeta de identidad, cuando cumplen los 18 años automáticamente el sistema les exige el nuevo documento, y en algunas oportunidades los estudiantes se demoran u olvidan realizar este trámite, entonces el sistema les suspende la TMC hasta tener cumplido este requisito.
Dicho sea de paso que, para el ii-2015, 147 estudiantes tuvieron la TMC suspendida y, aunque ya se actualizaron los documentos, están en espera de la recuperación de los recursos económicos que tenían "en espera".

\section{El fenómeno de simultaneidad}

El PJEA apoya también a estudiantes que han iniciado su formación en el sEna y si ingresan a la Universidad se les respeta su cupo para las TMc. Para ello, es muy importante manejar de manera adecuada el tema de simultaneidad, es decir, que no aparezcan al mismo tiempo en las dos entidades. Es preciso que las dos instituciones hagan la respectiva verificación y se priorice la institución donde el nivel de educación sea mayor, para recibir un beneficio más completo.

\section{Funcionamiento del programa JEA en la Uni- versidad de la Amazonia}

En la actualidad, para el funcionamiento del PJEA en la Uniamazonia, se manejan tres plataformas de información denominadas Sistema Integrado para Jóvenes en Acción-sija, las cuales funcionan en la oficina de la OARI; aquí se les hace el preregistro y el estudiante continúa con el trámite de manera independiente, los estudiantes son los encargados de subir los documentos, en formato pdf; una vez hecho el pre-registro, y haciendo uso de la línea gratuita del programa y la página web de la Universidad de la Amazonia en el link JEA, los estudiantes envían la documentación y posteriormente reciben un email, cuyo resultado puede ser: 1) el proceso fue exitoso, 2) el documento tuvo algún problema o invalidez, o 3) se identificaron problemas de codificación.

Además de los pasos para el registro, en dicho link, el estudiante encuentra información actualizada acerca de las fechas de los talleres, la caracterización dentro de cambio de documento y por supuesto las fechas y procedimiento para hacer efectivas las transferencias.

Una vez que el estudiante ha sido aceptado en el programa y están realizados los talleres de capacitación, se sigue con el proceso de "bancarización". La bancarización consiste en que a cada estudiante se le hace entrega de una tarjeta para que efectúe los retiros de su dinero, este proceso se realiza un par de semanas antes de la llegada del recurso. Si bien la tarjeta es la que va a acompañar al estudiante durante todo el proceso, el pago a los beneficiarios del programa está condicionado a fechas "pico y placa" para los retiros, ya sea por tarjeta o por ventanilla. Lo anterior no con el 
ánimo de no congestionar los pagos, sino que se hagan en los días establecidos; esto exige gran cuidado pues si el estudiante no realiza el cobro del dinero en las fechas establecidas, este es devuelto al fisco nacional. Las causas más comunes de porqué los estudiantes no cobran sus recursos en las fechas establecidas están asociadas a que las fechas de entrega coinciden con días agendados para prácticas de campo de algunos estudiantes, razón por la cual no pueden retirar el dinero. Es por lo anterior que se definió dar dos días más de reposición.

\section{Responsabilidades de la Universidad y el DPs}

Cuando se suscribió el convenio entre las dos instituciones, se indicó que se iba a tener una persona a cargo de la supervisión y el manejo del programa dentro de la Universidad, un enlace DPS- Universidad.

La persona encargada por la Universidad, en este caso, tiene como funciones realizar las preinscripciones de los estudiantes, estar pendiente de los talleres, enviar de manera periódica y cumplida los reportes de las bases de datos tanto de los matriculados, como de los de permanencia y publicar toda la información pertinente que provenga del DPS en lo que tiene que ver con el manejo de los requerimientos que hace la institución aliada, por ejemplo, por simultaneidad o cambio de documento.

La persona del DPS que realiza el enlace realiza la función de subir los documentos a la plataforma cuando esta está congestionada a través del mecanismo de ingresos de documentos por el link de novedades; de igual forma, es la persona encargada de orientar y dar respuesta a las diferentes preguntas y reclamaciones que presentan los beneficiarios y aspirantes el PJEA. Por otra parte, el DPS también es el encargado de velar por el buen funcionamiento y manejo de la plataforma sija.

\section{Metodología}

\section{Localización y caracterización de la población objeto del estudio}

La Universidad de la Amazonia se encuentra localizada en el municipio de Florencia, departamento del Caquetá, al suroccidente colombiano. Según el DANE (2014), el municipio cuenta con 169336 habitantes, de los cuales el 28,4\% son categorizados como jóvenes.

En términos de formación profesional, existen dos instituciones públicas que ofrecen este servicio que son: la Universidad Nacional Abierta y a Distancia (UNAD) y Universidad de la Amazonia. Específicamente, la
Universidad de la Amazonia alberga 8093 estudiantes, con periodicidad semestral de ingresos promedio de 800 estudiantes (Consejo Académico de la Universidad de la Amazonia, 2015). No obstante, también mantiene un ingreso constante de estudiantes procedentes de diferentes partes del país y, precisamente, parte de estos estudiantes son beneficiarios del Programa Jóvenes en Acción del Departamento para la Prosperidad Social (OARI, 2015)

Así las cosas y con el ánimo de evaluar la ejecución de este programa desde los actores, el presente estudio abordó dos tipos de actores: los institucionales y los beneficiarios del programa.

Los institucionales son las que ejecutan el programa en el orden departamental -DPs y el enlace-, en este caso, las directivas de la Universidad encargadas para este. De manera paralela, se abordaron los beneficiarios del programa - los jóvenes en acciónen este caso, beneficiarios del programa que estudian en la Universidad de la Amazonia y que habían recibido el apoyo para desarrollar sus estudios de educación superior.

\section{El enfoque metodológico}

La presente investigación siguió los lineamientos del paradigma de investigación crítico-social de la escuela de Frankfurt, trabajando por generar reflexiones que permitan la emancipación del ser humano, que considera el saber cómo un tipo de conocimiento crítico en las personas, en donde la ciencia, el conocimiento y la educación son vistos como herramientas fundamentales del cambio social.

\section{El método}

El diseño metodológico de la investigación involucró una metodología cualitativa, el método empelado fue el análisis de conversaciones y la técnica de producción de los datos en este caso fue la entrevista interpretativa y el análisis de documentos (Sauto, Boniolo, Dalle y Elbert, 2005). A continuación, se describe al detalle el proceso metodológico.

\section{Población y muestra}

Caracterización del Programa Jóvenes en Acción en la Universidad de la Amazonia

El proceso de investigación inició con la revisión de la información secundaria disponible acerca del 
programa objeto de estudio, para ello se acudió a una rigurosa revisión de la información disponible en línea, en la página web del Departamento para la Prosperidad Social (DPS). Posteriormente, se tuvo acceso a diferentes documentos en físico y que son de carácter público del programa; así como las bases de datos, informes técnicos y registros de la ejecución del mismo. De esta manera, la técnica de levantamiento de la información empleada fue el arqueo de archivo y para la sistematización se emplearon plantillas de doble entrada donde se iba registrando el nombre del documento, la localización, el hallazgo de interés, la página y una columna de observaciones. Las variables de interés fueron identificadas y analizadas desde lo general - a nivel nacional- a lo particular - caso Caquetá-. Dichas variables objeto de la pesquisa fueron: antecedentes, objetivo del programa, metodología general de operación, componentes que lo integran, cobertura programada y efectuada y resultados obtenidos por el programa durante el 2014.

\section{Percepción social de los jóvenes y encargados del programa acerca del desarrollo de este}

Para poder conocer a profundidad, por un lado, la operatividad del programa Jóvenes en Acción en el departamento del Caquetá, esencialmente en el municipio de Florencia, así como los ajustes metodológicos que sobre la marcha se tuvieron que hacer, los retos y desafíos que se enfrentaron, y por otro lado para poder indagar qué tanto los beneficiarios conocen el programa y su lectura acerca de la operatividad del mismo, se acudió a la entrevista como instrumento para el levantamiento de la información abierta. Cada entrevista fue grabada y transcrita buscando mantener la originalidad de lo expresado por el entrevistado. Posteriormente, la grabación convertida en texto fue llevada al software de procesamiento Atlas.Ti, y codificada, estableciendo categorías de análisis y familias de categorías, lo cual permitió tener claridad sobre la percepción del entrevistado acerca del programa. Finalmente, se hizo la triangulación de la información.

\section{Resultados y Discusión}

\section{Caracterización y resultados del PJEA en la Universidad de la Amazonia.}

La educación es tan importante para nuestro país que está consagrada de manera directa, indirecta y transversal en la Constitución Política de Colombia (1991), muchos de sus apartados se refieren a la educación en múltiples aristas, al punto de ser consagrada como un derecho fundamental de los niños en el art. 44, un derecho de la persona y un servicio público en el art. 67, por mencionar algunos acápites, pues el tema es bastante generoso y tiene implicaciones del orden económico, administrativo, social, cultural y demás. Manrique (2009) expresa que la educación como un asunto público debe ser de interés colectivo, toda vez que su impacto se reflejará en la sociedad y el Estado en general. Igualmente, sostiene que el papel del Estado es garantizar la inversión de recursos y el despliegue institucional apropiado, con el propósito de que la educación cumpla con su filosofía de formación de auténticas personas-ciudadanos y una garantía del progreso social equitativo.

Así las cosas, las erogaciones producidas por la educación deberán ser concebidas como una inversión para el desarrollo integral de la sociedad, la construcción de un mejor proyecto de país y la generación de motores de desarrollo académico y evolución científica. La Organización Europea para la Cooperación Económica (2012) plantea que la inversión de recursos en educación contribuye al crecimiento económico y al desarrollo social; expone que estas inversiones garantizan condiciones de calidad para los educandos, que podrán desarrollar competencias de alto nivel, sin encontrar en sus condiciones socioeconómicas, de género, étnicas o familiares un obstáculo. Aduce que la probabilidad de tener un rendimiento bajo se duplica en estudiantes de condiciones socioeconómicas desfavorables. Igualmente, plantea que la inversión en educación, además de mejorar las condiciones humanas del individuo, mejora las perspectivas laborales, incrementa los participantes del sistema económico y genera menor dependencia a las ayudas públicas, convirtiendo entonces a la inversión en la educación en un factor de desarrollo futuro para la sociedad. En este sentido, el Banco Mundial (2008) sostiene que, en Colombia, se han generado algunos importantes programas sociales para el fomento de la educación, entre ellos, el de Familias en Acción, el Programa de Ampliación de Cobertura de la Educación Secundaria (Paces), hoy clausurado, el subsidio a escuelas privadas para la prestación del servicio a estudiantes de bajos ingresos, entre otros.

$\mathrm{El}$ anterior panorama permite evidenciar que el fomento a la educación en Colombia ha sido pensado más allá de una simple inversión para el sostenimiento de las instituciones, el pago de los maestros y la 
adquisición de materiales para el desarrollo de las actividades académicas. Es claro que el Gobierno nacional ha dimensionado a la educación como un conjunto integral, donde la calidad de vida de los estudiantes tiene alta incidencia en su desarrollo educativo. En este sentido, Melo, Ramos y Hernández (2014), luego de realizar un recorrido por el panorama de la educación en Colombia, ratifican que existe una estrecha relación entre los logros académicos y las variables socioeconómicas, y que muchos de los esfuerzos de mejoramiento de las instituciones de educación superior (IES) se ven difuminados por la influencia de estas condiciones en sus estudiantes; plantea que las medidas de política del Estado y las instituciones relacionadas, entre otros, deberán promover estrategias que permitan contrarrestar el impacto negativo derivado de las condiciones socioeconómicas de los estudiantes.

Es por ello que el Gobierno nacional, a través del Ministerio de Educación Nacional (MEN) sus departamentos administrativos, programas sociales y demás, ha generado un sin número de estrategias para buscar garantizar la permanencia de los estudiantes en sus aulas de clase; prueba de ello son, según el men (2010), los programas de inversión en transporte escolar, inversión en el sector solidario, alimentación escolar, gratuidad educativa, articulación con la Red para la Superación de la Pobreza Extrema Juntos (Red Juntos) y articulación con programa Familias en Acción, los cuales clasifica como estrategias de apoyo económico y apoyo complementario a la canasta escolar.

Los esfuerzos por mejorar las condiciones de la educación y de los estudiantes mismos han traslapado la educación básica y media. En la actualidad, existen grandes iniciativas del ejecutivo nacional por garantizar óptimas condiciones en la educación superior, programas como los créditos educativos y de sostenimiento del Icetex, apoyos a la universidades para la retención estudiantil, la asignación de diez mil becas y el anuncio de once mil para la próxima vigencia, adición de recursos para los presupuestos globales de las universidades (CREE, entre otros) y la implementación de programas como Jóvenes Rurales y Jóvenes en Acción, son una clara muestra del interés por la cualificación del servicio educativo, especialmente en lo que se refiere a la retención estudiantil, el incremento de la cobertura y la educación de calidad. Todo apunta a que el Gobierno busca, tal y como lo afirma el eslogan de la actual administración nacional, la construcción de un nuevo país a través de paz, equidad y educación. Estas estrategias parecen apuntar a lo planteado por Marchesi (2009), que sostiene que, en el proyecto "Metas educativas 2021: la educación que queremos para la generación de los bicentenarios" el cual fue formulado por la Organización de Estados Iberoamericanos para la Educación, la Ciencia y la Cultura (oei) junto con la Conferencia Iberoamericana de Ministros de Educación - busca potencializar a la educación como una fuerza transformadora de las sociedad en situación de pobreza y desigualdad, planteamientos que están en sintonía con la filosofía de intervención estatal al sector educación.

Ahora bien, para nuestro programa objetivo de estudio, denominado Jóvenes en Acción, el cual fue pensado como una estrategia del Gobierno nacional, según el DPS (2013), de transferencias monetarias condicionadas hacia la formación para el trabajo, la generación de ingresos autónomos y el mejoramiento de las condiciones de vida de los jóvenes en situación de pobreza. Las motivaciones para la implementación del programa, entre otras, son las fallas del mercado laboral, los bajos índices de formación académica de la población vulnerable, los altos niveles de deserción causados por la ausencia de recursos y las difíciles condiciones económicas de los padres/responsables; situaciones que de no ser atendidas de manera oportuna, según la Universidad Nacional (2009), privan a la sociedad de contar con capital humano capacitado para mejorar la calidad de vida familiar y generar desarrollos en materia económica, social y académica. Al respecto, el MEN (2009) sostiene que estos escenarios están siendo contrarrestados con el proyecto de Revolución Educativa, el cual busca garantizar una educación de calidad, responder a las expectativas de los colombianos y enfrentar los desafíos del mundo contemporáneo. Así las cosas, es evidente que el programa Jóvenes en Acción va más allá de una simple transferencia de recursos, su génesis es garantizar la permanencia de los estudiantes en las IEs, materializando en gran medida las intencionalidades del Ministerio de Educación y el Gobierno nacional.

Este programa, para departamentos como el Caquetá, donde se tienen altos índices de desempleo, múltiples víctimas de los conflictos sociales, una economía debilitada y extractiva, reducidos escenarios de formación y minúsculas oportunidades para los jóvenes, se convierte en una oportunidad para garantizar un apoyo económico a quienes han ingresado a la educación superior que les permita contribuir a su permanencia y culminación del proceso de formación profesional. Para la Universidad de la Amazonia, entidad del orden estatal, creada según la Ley 60 de 1982 , este programa se ha convertido en un aliado estratégico y un gran benefactor de los educandos. 
Para nuestro caso de estudio, la implementación del programa Jóvenes en Acción se materializó en el primer semestre del 2014, a través del convenio suscrito entre la Universidad de la Amazonia y el Departamento de la Prosperidad Social (DPS). En el primer semestre de ejecución se reportaron 1391 beneficiarios, los cuales representaban el 18,63 \% de la población estudiantil de la Universidad, pese a la restricción existente para el ingreso al programa.

Para el siguiente semestre (II-2014), el programa sufrió un significativo aumento, pasando de 1391 estudiantes a 2163 , los cuales pasaron a representar el $28,96 \%$ de la población estudiantil, convirtiéndose en una comunidad representativa en la Universidad.
Finalmente, para el primer semestre del 2015, se llegó a beneficiar a 2223 estudiantes, lo que representó el 27,74\% de la población estudiantil.

La disminución porcentual obedece al gran número de estudiantes que se vincularon en primer semestre, especialmente a los programas de tecnologías, campus diferentes al Caquetá y educación a distancia, los cuales no tienen posibilidad de acceder a los beneficios del programa.

Todos los programas de pregrado - activos y propios- reportaron beneficiarios, para ese momento la Universidad contaba con 20 programas de pregrado, 16 del nivel profesional y 4 del nivel tecnológico, con los siguientes beneficiarios:

Tabla 1

Beneficiarios del PJEA por programa y facultad

\begin{tabular}{|c|c|c|c|}
\hline \multirow{2}{*}{ Facultades } & \multirow[b]{2}{*}{ Beneficiarios } & \multicolumn{2}{|c|}{ Representación \% } \\
\hline & & Por facultad & En la universidad \\
\hline Facultad de Ciencias Contables, Económicas y Administrativas & 534 & $100 \%$ & $24,0 \%$ \\
\hline Programa de Contaduría Pública & 313 & $58,6 \%$ & $14,1 \%$ \\
\hline Programa de Administración de Empresas & 221 & $41,4 \%$ & $9,9 \%$ \\
\hline Programa de Administración Financiera - Distancia & 0 & $0,0 \%$ & $0,0 \%$ \\
\hline Programa de Tecnología en Gestión de Mercados (inactivo para el II-2014) & 0 & $0 \%$ & $0,0 \%$ \\
\hline Facultad de Ingenierías & 496 & $100 \%$ & $22,3 \%$ \\
\hline Programa de Ingeniería Agroagrícola & 277 & $55,8 \%$ & $12,5 \%$ \\
\hline Programa de Ingeniería de Sistemas & 146 & $29,4 \%$ & $6,6 \%$ \\
\hline Programa de Ingeniería de Alimentos & 73 & $14,7 \%$ & $3,3 \%$ \\
\hline Programa de Tecnología en Informática y Sistemas - Distancia & 0 & $0,0 \%$ & $0,0 \%$ \\
\hline Facultad de Ciencias Básicas & 193 & $100 \%$ & $8,7 \%$ \\
\hline Programa de Química & 65 & $33,7 \%$ & $2,9 \%$ \\
\hline Programa de Biología & 128 & $66,3 \%$ & $5,8 \%$ \\
\hline Facultad de Derecho y Ciencias Políticas & 270 & $100 \%$ & $12,1 \%$ \\
\hline Programa de Derecho & 270 & $100 \%$ & $12,1 \%$ \\
\hline Tecnología en Criminalística & 0 & $0,0 \%$ & $0,0 \%$ \\
\hline Facultad de Ciencias Agropecuarias & 245 & $100 \%$ & $11,0 \%$ \\
\hline Programa de Medicina Veterinaria y Zootecnia & 245 & $100 \%$ & $11,0 \%$ \\
\hline Facultad de Ciencias de la Educación & 485 & $100 \%$ & $21,8 \%$ \\
\hline Licenciatura en Matemática y Física & 113 & $23,3 \%$ & $5,1 \%$ \\
\hline Licenciatura en Inglés & 220 & $45,4 \%$ & $9,9 \%$ \\
\hline Licenciatura en Lengua Castellana & 64 & $13,2 \%$ & $2,9 \%$ \\
\hline Licenciatura en Ciencias Sociales & 88 & $18,1 \%$ & $4,0 \%$ \\
\hline Licenciatura en Educación Artística - Convenio con Surcolombiana & 0 & $0,0 \%$ & $0,0 \%$ \\
\hline Licenciatura en Pedagogía Infantil - Distancia & 0 & $0,0 \%$ & $0,0 \%$ \\
\hline Programa en Tecnología en Salud Ocupacional - Distancia 0 & $0,0 \%$ & $0,0 \%$ & \\
\hline TOTAL BENEFICIARIOS & 2223 & $\mathrm{n} / \mathrm{a}$ & $100 \%$ \\
\hline
\end{tabular}

Nota. Elaboración propia basada en datos de Oficina Asesora de Relaciones Interinstitucionales -Uniamazonia. 
Tal y como se evidencia en la tabla 1, las facultades con mayor índice de participación son Ciencias de la Educación, Ingenierías y Ciencias Contables, Económicas y Administrativas, fenómeno que obedece a que son las que cuentan con mayor número de programas académicos. Son las facultades con mayor demanda en la región, y con excepción de Ingeniería, la oferta de programas en jornada diurna y nocturna. La vigencia 2014, en palabras de los coordinadores del convenio, superó las expectativas y proyecciones, pues se logró prácticamente triplicar el número de cupos presupuestados. Alrededor del programa, se generó todo un sistema social, pues las TMC se convirtieron en una oportunidad para mejorar las condiciones de permanencia, máxime cuando cerca del $80 \%$ de la población atendida por la Universidad se encuentra en los estratos socioeconómicos 1 y 2 . El programa, además de ser benefactor para los estudiantes, se convirtió rápidamente en un aliado estratégico para la universidad en el cumplimiento de su actuar misional, la generación de bienestar universitario y la constante lucha por combatir, por ejemplo, las realidades críticas planteadas por la Unesco (2004), respecto a las desigualdades crecientes en la educación, la discriminación, la exclusión de procesos de educación de calidad para todos; los impactos del convenio, en palabras de la Unesco, se pueden clasificar como un acercamiento a una "cultura de igualdad".

No obstante, pese a las políticas de flexibilización, acercamiento y acompañamiento realizadas por los ejecutores del convenio, la permanencia de los estudiantes está determinada básicamente por el esfuerzo que ellos impriman durante su semestre académico, escenario en el cual deben mantener un promedio mínimo de calificación, y aunque para muchos intelectuales de la educación, la valoración cuantitativa debe ser relegada a un segundo plano, dentro de los términos administrativos del convenio, se exige el cumplimiento del promedio mínimo (3.0) como requisito habilitante. Esta situación generó que de 1391 que iniciaron su participación en el PJEA en el 2014-I, 925 quedaran activos, 461 condicionados y 5 inactivos. De los 2163 que iniciaron su participación en el programa en el periodo 2014-2, a cierre del semestre, 1623 quedaron en estado activo, 590 quedaron en estado condicionado y 6 en estado inactivo. En el periodo 2015-I, se beneficiaron 2223 estudiantes, de los cuales a cierre de semestre 1888 quedaron en estado activo, 543 condicionados y 7 inactivos.

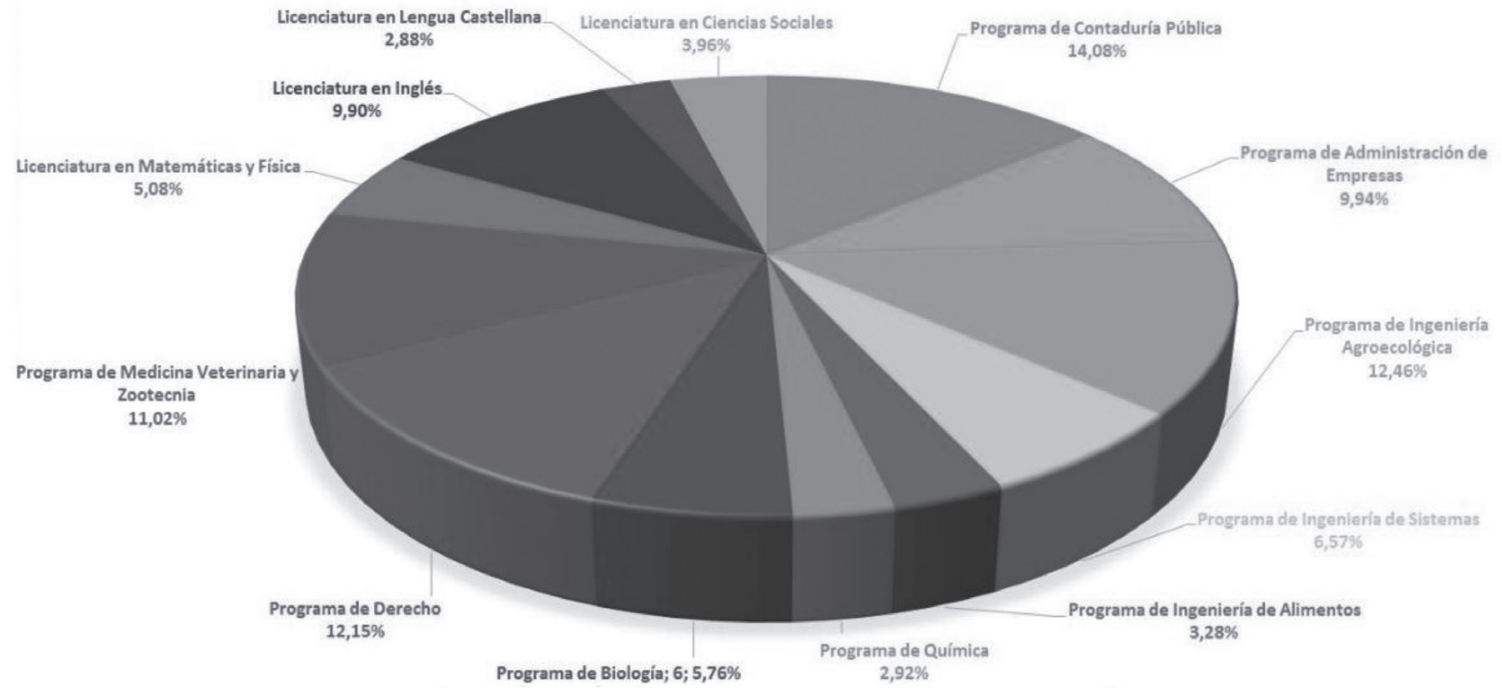

Figura 1. Participación porcentual de los programas de pregrado en el pjea. Elaboración propia. 


\section{Percepción social de los encargados del PJEA acerca del programa}

Cifuentes, Montenegro y González (2013) exponen que los resultados esperados por los programas de acompañamiento y apoyo a los estudiantes, por lo general, están alrededor de asegurar las condiciones mínimas requeridas para fomentar un adecuado proceso de aprendizaje, desarrollo personal y profesional, y el fortalecimiento de los proyectos de vida. En este sentido, la percepción social de los encargados del programa acerca de la ejecución de este permitió identificar alrededor de 20 categorías de análisis relacionadas con el ejercicio de las Transferencias Monetarias Condicionadas (TMC) como un apoyo que otorga el Gobierno nacional a los jóvenes, entendidas como una estrategia que refleja el impacto en los beneficiarios a juicio de los ejecutores del programa alrededor de categorías como "empeño", "compromiso", "atención" e "interés". El empeño reflejado en un compromiso con sus ingresos de créditos académicos, lo cual ha generado mayor atención a los calendarios de notas, ingresos, capacitaciones y demás información de interés; lo anterior se pone en evidencia al emplear con mayor frecuencia las TIC, visitar el sitio web, el link y estar más atentos al correo electrónico.

Silva (2011) expresa que una de las etapas más complejas en la educación superior está ligada a la transición e integración de los jóvenes al sistema universitario; además, reconoce que las desventajas socioeconómicas y culturales, las cuales se acentúan en la población vulnerable, obstaculizan un buen desempeño académico. Es por esto que los encargados del programa afirman que invierten gran parte de su tiempo libre, sin beneficios económicos adicionales, en el perfeccionamiento de su labor. Igualmente, reconocen que el éxito del programa se debe a la "comunicación eficiente" y al acceso a la "información", lo que favorece la permanencia y el interés en el mismo. También consideran que conforme se ha avanzado, el programa y los canales de comunicación han sido más eficientes y se ha disminuido el "índice de pérdida del beneficio".

Las TMC, a juicio de los ejecutores han contribuido al "mejoramiento académico", pues los estudiantes se esmeran por mantener un buen promedio y ganarse la bonificación "premio", que consiste en \$200 000 adicionales al final del semestre académico. A pesar de los logros anteriores, uno de los limitantes del programa, es que no cubre los pregrados en "Modalidad a distancia" $y$, en esta modalidad, los estudiantes requieren de realizar desplazamientos cada fin de semana, pues son jóvenes rurales o de los pueblos, quienes además de viajar, deben pernoctar y alimentarse en la ciudad. Además de lo anterior, existe una gran tensión entre los ejecutores del programa y los actuales aspirantes, pues aunque se han recibido las solicitudes de ingreso, no es claro si se podrá ampliar la cobertura para el próximo año. De esta manera, la Universidad y el DPs tienen, por un lado, la

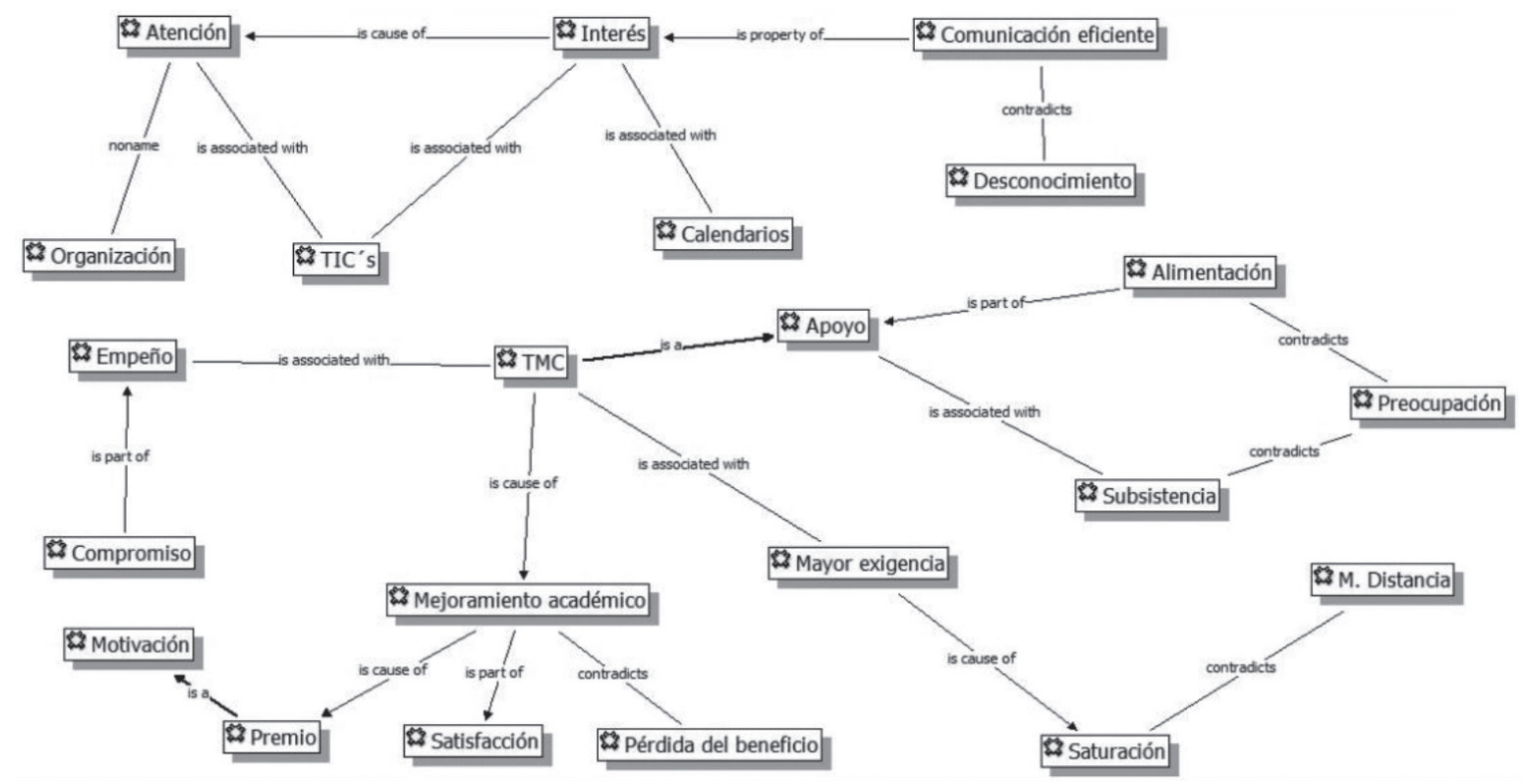

Figura 2. Categorías de percepción por parte de los encargados del PJEA en la Uniamazonia. Elaboración propia. 
"satisfacción" de los resultados logrados por el programa y, por el otro, la "preocupación", porque la disponibilidad de cupos para nuevos beneficiarios es incierta. De igual forma, de llegar a ampliarse la cobertura bajo las actuales coyunturas económicas del país, se auguran reingenierías del programa en torno a los niveles de exigencia académicos y los requisitos administrativos, en tal sentido aparecen categorías como "mayor exigencia", lo cual exige una nueva forma de "organización".

En la figura 2, se pueden apreciar las categorías de análisis identificadas en las percepciones de los ejecutores del programa.

\section{Imaginarios de los estudiantes alrededor del PJEA acerca del programa}

La Comisión Europea (2015) sostiene que su programa Juventud en Acción tiene como base dos dimensiones: la "participación" y la "empleabilidad"; la primera busca generar en los jóvenes la inclusión social y la solidaridad; la segunda busca la generación de oportunidades en educación y el mercado laboral. De esta manera, el programa busca nutrir de jóvenes activos el sistema social, generando altos niveles de educación y habilidades, adaptabilidad y creatividad, mercados laborales inclusivos y participación activa en la sociedad. La filosofía de este programa, la cual guarda estrecha relación con la del PJEA, se difumina al caracterizar el imaginario de los estudiantes, toda vez que estos perciben las тсм como un estímulo para su proceso de formación y el cumplimiento de su meta académica, desconociendo de esta manera las intencionalidades secundarias del ejecutivo nacional que, para el caso, están relacionadas directamente con la generación de agentes activos para el desarrollo económico del país.

Esta situación es contraria a las percepciones encontradas por DNP (2004), - tal vez por el nuevo enfoque y modelo de ejecución-, donde los beneficiarios del programa "Jóvenes en Acción - Capacitación laboral de jóvenes desempleados de bajos recursos (JEA)" sostienen que los beneficios del programa están relacionados con adquirir conocimientos para el trabajo, certificación de sus competencias, mejoramiento personal, entre otros, claro está, sin desconocer el impacto positivo del apoyo económico, sin embargo, este no se convirtió en el eje exclusivo del programa.

Dentro de las expresiones de los estudiantes acerca del programa, se identificaron en 47 categorías de análisis correspondientes a las familias denominadas: proceso, destino de las transferencias y cambio.

\section{El proceso}

Aparece asociado con códigos de expresión tales como: "dificultades", "repetición", "información", "esperanza", "desconocimiento", "no ilusión", "varios intentos", "base de datos incompleta", "cambio del Sisben", "desinformación", "apoyo en el proceso", "aplazamiento del pago", "retrasos", "documentación incompleta", "estudiantes foráneos", y "otros programas". Los beneficiarios del PJEA entrevistados reconocen que se enteraron del programa gracias a la campaña de socialización y la "información" entregada cada semestre por parte de la Universidad, la cual aparece como una contradicción con el "desconocimiento", que sobre todo se refleja en los estudiantes de los primeros semestres, los cuales argumentan que en el colegio nunca se les habló del programa, lo cual podría ser una factor que limita el interés de la media por ingresar a un escenario de educación superior.

Una vez conocido el programa, lo percibieron como una "esperanza" para poder mejorar sus condiciones, sobre todo para quienes ya estaban estudiando en un semestre avanzado cuando llegó el PJEA. Así las cosas, con la información y la esperanza, aparecen de manera contradictoria las "dificultades" en el proceso, sobre todo porque consideran que como estudiantes no eran juiciosos a la hora de verificar los requisitos y aunque reconocen el "apoyo en el proceso", generalmente el puntaje del Sisben era muy alto, o no se anexaban los documentos como debían ser. Esto aparece como la causa principal de los "aplazamientos de los pagos", los "retrasos" o los "rechazos" para el ingreso. Los "rechazos" aparecen asociados principalmente a que los documentos que debían subir a la plataforma, no se hacían en el formato solicitado y se sobrepasaba el límite de Kb permitido, o también el puntaje de la ficha del Sisben era muy alto. A pesar de los rechazos, algunos estudiantes repitieron el proceso, por ello aparece la "repetición", como una "esperanza".

Por su parte, las causas de los "aplazamientos de los pagos" están relacionadas con su juicio por el cambio de lugar de residencia de departamento "estudiantes foráneos", el cambio de tarjeta de identidad a cédula de ciudadanía "documentación incompleta" o también que se les olvidaba el día del pago, o que aparecen por simultaneidad en otros programas. En la figura 3, es posible diferenciar las categorías y los ejes de tensión que los entrevistados, beneficiarios del programa, dejaron ver durante el proceso: 


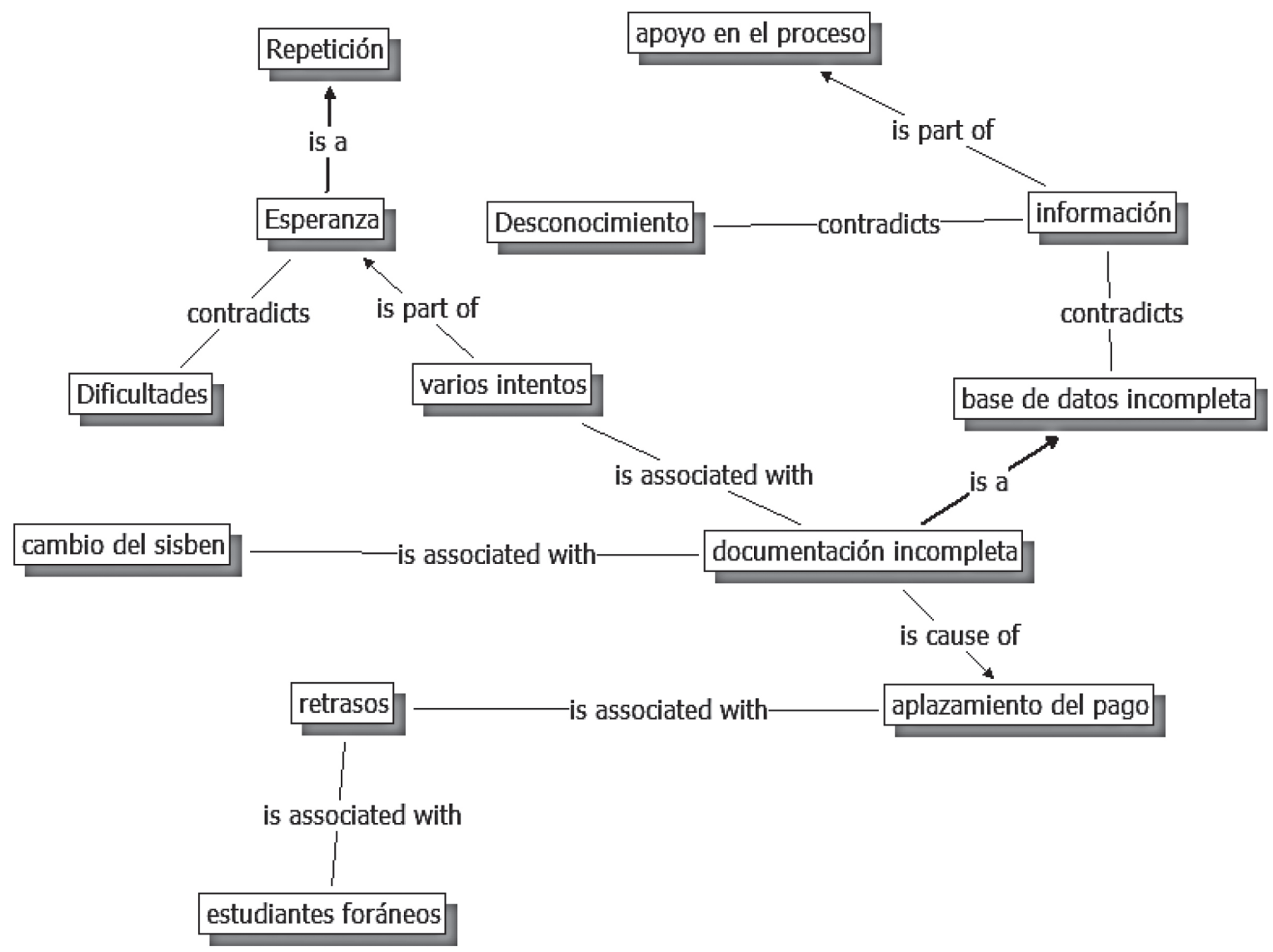

Figura 3. Familia de percepciones "el proceso". Elaboración propia.

\section{El destino de las transferencias}

Según el DNP (2008), los apoyos económicos girados por el programa "Jóvenes en Acción Capacitación laboral de jóvenes desempleados de bajos recursos (JEA)" pretendían cubrir los costos inherentes a transportes y alimentación durante la fase lectiva y de práctica. Este programa propendía a la inserción laboral y básicamente buscaba el entrenamiento del talento humano para que se sometiera a procesos de reclutamiento organizacional y, por ende, todas las ayudas percibidas por los beneficiarios se asimilaban en este sentido, a contrario sensu, los entrevistados dejaron ver desde su lado más sincero que las TMC están asociadas con categorías como "apoyo", "complemento" y "ayuda", algo sin lo que, si no llegara a seguirse dando, podrían seguir estudiando pero en condiciones asociadas con la "dificultad". Aparecen los "padres de familia" como el principal apoyo económico para la realización de los estudios.
Al consultar los destinos de las TMC, las categorías halladas fueron: "transportes", "matrícula", "necesidades básicas" y "prácticas de campo". Los consultados dejaron en este tema en particular expresiones como: "yo así ya no soy tanta carga para mis padres", "yo pude comprar un mejor colchón donde dormir", "ya no tengo la presión de que debo conseguir un trabajo", "no sé qué haría pues mi familia tiene una situación económica difícil". Sin embargo, y pese al juicioso ejercicio de algunos, aparecen de manera contradictoria elementos como "despilfarro", "rumba" y "regalos", sobre todo, en las primeras transferencias, lo cual fue cambiando con el tiempo (figura 4).

Las TMC han permitido establecer una relación diferencial en algunos casos padres-hijos, los hijos considerando que alivian una carga a sus padres, así no sea con la matrícula sino con el mejoramiento de las condiciones de vida para estudiar: alimentación, hospedaje, transportes y materiales, entre otras. 


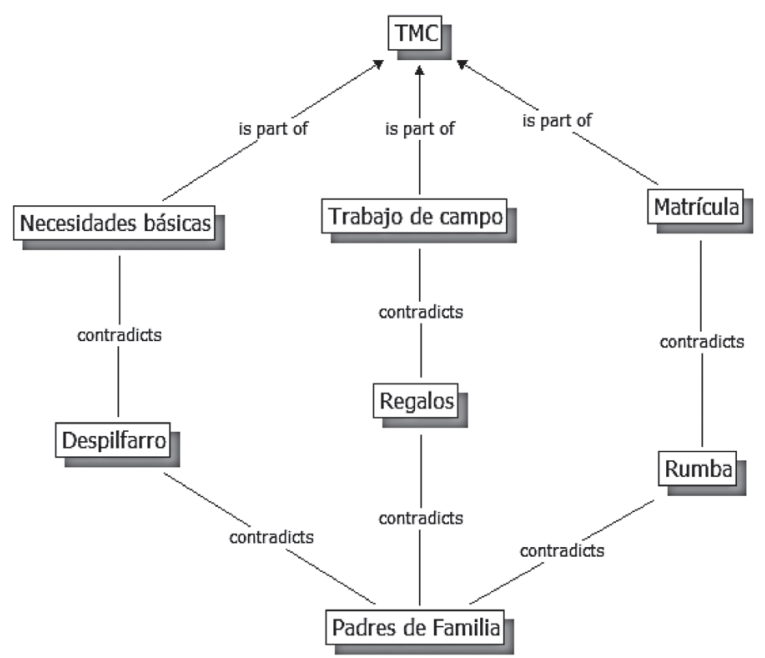

Figura 4. Destino de las TMC. Elaboración propia.

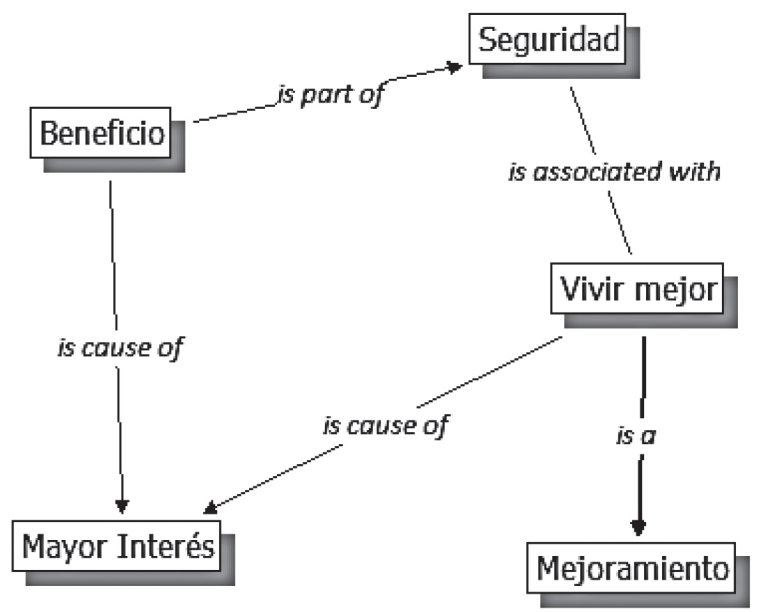

Figura 5. Percepción de los estudiantes alrededor del programa Familia cambio. Elaboración propia.

\section{El cambio}

Según López, Espinoza, Rojo, Flores y García (2015), los principales gastos de los estudiantes universitarios están relacionados con materiales, artículos personales, alimentación fuera de casa, transporte, servicios tecnológicos, entre otros. Estas necesidades son básicas y constantes en el proceso de formación, pues obedecen a dinámicas mismas de la Universidad, ahora bien, para los estudiantes de la Universidad de la Amazonia que se benefician del PJEA, la posibilidad de contar las TMC permite cubrir estos gastos e incluso generar algunos excedentes para actividades de ocio y diversión.
Los beneficiarios consultados ven claramente un antes y un después de ser beneficiarios del programa, la categoría que más los identifica es la de "seguridad", entendida como la tranquilidad que genera un ingreso adicional para garantizar su complemento de gastos al estudiar, lo cual se identifica asociado con un "cambio para vivir mejor" y una lectura a sus compañeros de "beneficio", "mejoramiento" y "mayor interés" (figura 5).

En términos generales, evalúan el programa como exitoso, beneficioso, importante y no complejo de acceder y mantener, un complemento que si bien no define si se estudia o no, pero se es consciente de que sin él las condiciones para estudiar desmejorarían notablemente.

\section{Principales retos}

Los retos del PJEA están relacionados especialmente con la cobertura. Cada día son más los jóvenes que presentan solicitudes de ingreso y menos los que pueden ingresar. A la fecha, solo está asegurado el sostenimiento de los estudiantes que ingresaron en los periodos 2014 y 2015-I, lo demás es incertidumbre. Todo esto depende cien por ciento de la capacidad del Gobierno nacional de disponer más recursos para el programa.

Los requisitos para el ingreso al programa resultan bastantes generalizados y son muy incluyentes; de esta manera, cualquier persona que aplique con unos requisitos mínimos ingresa. De igual forma, el sostenimiento exige un promedio mínimo para el sostenimiento que implica lograr un 3,0. Este es el mínimo aprobatorio, lo cual hace que su nivel de exigencia sea el mínimo. Uno de los retos que enfrenta el programa es cómo hacer que el PJEA tenga un nivel de exigencia mayor para el sostenimiento y, de esta manera, sea más valorado.

Otro de los retos que enfrenta el programa son las altas demandas en los programas de formación en modalidad a distancia. Estos estudiantes son generalmente los de más bajos recursos, que provienen de lugares distintos a Florencia y deben trabajar y la única opción es estudiar los fines de semana en modalidad a distancia. Esta población, si bien tiene generalmente un ingreso producto de su trabajo entre semana, debe asumir costos adicionales como transportes, comidas fuera de casa y alojamientos.

\section{Conclusiones}

Una vez evaluado el pjeA en la Universidad de la Amazonia, se puede concluir que el programa ha sobrepasado las metas establecidas, que el número de beneficiarios está muy por encima de lo que se 
presupuestado inicialmente, y que el impacto hoy llega al 28,06\% de la población estudiantil.

Si bien es un apoyo interesante, a juicio de los beneficiarios, las TMC son un complemento a sus otros ingresos y sus condiciones de vida para mantenerse en el proceso educativo han mejorado considerablemente. Sin embargo, por la laxitud de los requisitos, en términos de promedio, cualquier persona que pierda el beneficio puede volver a ingresar al programa.

El imaginario de los estudiantes alrededor del PJEA es bastante positivo y denota esperanza, motivación e ilusión como fundamento para mejorar y salir adelante. Por su parte, los responsables del programa han logrado establecer lazos de unión y afecto hacia los estudiantes beneficiarios de este, expresado a través del acompañamiento permanente, su mayor preocupación en el momento es el sostenimiento del mismo.

La política de beneficio económico del Gobierno para la educación superior, en el PJEA en particular, se ha convertido en un apoyo para los jóvenes que se interesan por acceder a un nivel profesional; sin embargo, los mecanismos de monitoreo y control son muy débiles, pues se adolece de un proceso de evaluación y selección riguroso para el sostenimiento en el programa, el cual vaya más allá de un mínimo cuantitativo, sino que involucre otros elementos que permitan de verdad fortalecer y premiar a quien más se lo merezca. En este sentido, es preciso recordar que, a nivel nacional, el apoyo a la permanencia estudiantil y la disminución de tasas de deserción en educación superior se ha dirigido especialmente al ofrecimiento de apoyos económicos y en menor medida a apoyos académicos.

\section{Referencias}

Banco Mundial. (2008). La calidad de la educación en Colombia: un análisis y algunas opciones para un programa de política. Unidad de Gestión del Sector de Desarrollo Humano Oficina Regional de América Latina y el Caribe.

Cifuentes S, A. Montenegro M. y. González, M. A. (2013). Evaluación del impacto de los programas de apoyo relacionados con la permanencia estudiantil en la Universidad Santo Tomás de la modalidad presencial de pregrado, Bogotá. Bogotá: Universidad Santo Tomas.

Comisión Económica Europea. (2015). Juventud en Acción, Guía para el Programa. Recuperado de http://www. juventudenaccion.injuve.es/opencms/export/download/formularios/Guiajea2013.pdf
Consejo Académico de la Universidad de la Amazonia. [CAUA]. (2015). Reporte de estudiantes admitidos II semestre 2015.

Constitución Política de Colombia. (1991). Derechos a la educación. Artículo 44 y 67.

Departamento para la prosperidad social. [DPs]. (2013). Manual operativo del Programa Jóvenes en Acción. Dirección del Ingreso Social. Bogotá: DPS.

Departamento Administrativo Nacional de Estadísticas. [DANE]. (2014). Caquetá en cifras. Recuperado de https://www.dane.gov.co/files/icer/2010/ caqueta icer_10.pdf

Departamento Nacional de Planeación. [dnp]. (2004). Análisis de resultados de las primeras convocatorias del programa Jóvenes en Acción. Informe Final. Bogotá, Colombia.

Departamento Nacional de Planeación. (DNP). (2008). Evaluación de políticas públicas, Subprograma Jóvenes en Acción: consultoría para la evaluación de impacto del Subprograma Jóvenes en Acción. Bogotá, Colombia.

Departamento para la Prosperidad Social. [DPs]. (2013). Programa Jóvenes en Acción. Recuperado de http:// www.urnadecristal.gov.co/gestion-gobierno/reg-strate-en-j-venes-en-acci-n-hasta-23-de-febrero.

López, M., Espinoza, A., Rojo, D., Flores, K. y García, A. (2015). Hábitos de consumo del estudiante universitario: el caso del Centro Universitario del Sur, de la Universidad de Guadalajara. Nova scientia, 7(13), 352-373. Recuperado de http://www. scielo.org.mx/scielo.php?script=sci_arttext\&pi$\mathrm{d}=$ S2007-07052015000100019\&lng=es\&tlng=es

Manrique, N. J. I. (2009). Protección constitucional del derecho a la educación y responsabilidad estatal por falla en el servicio de la educación. Tesis de Maestría en Derecho Administrativo, Facultad de Jurisprudencia. Universidad del Rosario, Bogotá, Colombia.

Marchesi, A. (2009). Las metas educativas 2021. Un proyecto iberoamericano para transformar la educación en la década de los bicentenarios. Revista Iberoamericana de Ciencia Tecnología y Sociedad, 12(4), 87-157. Recuperado de http://www.scielo.org.ar/scielo.php?script=sci_arttext\&pid=S1850-00132009000100007

Melo, L. A., Ramos, J. E. y Hernández, P. O. (2014). La educación superior en Colombia: situación actual y análisis de eficiencia. Borradores de Economía, 808, Bogotá: Banco de la República. Recuperado de http://www.banrep.gov. co/docum/Lectura_finanzas/pdf/be_808.pdf 
Ministerio de Educación Nacional. [MEN]. (2010). Apoyo económico. Recuperado de http://www.mineducacion. gov.co/1621/article-212402.html

Ministerio de Educación Superior. [MEN]. (2009). Deserción estudiantil en la educación superior colombiana, metodología de seguimiento, diagnóstico y elementos para su prevención, primera edición. Bogotá: Viceministerio de Educación Superior e Imprenta Nacional de Colombia.

OECD. (2012). Equity and Quality in Education: Supporting Disadvantaged Students and Schools. Paris: OECD Publishing.

Oficina de Relaciones Interinstitucionales. [OARI]. (2015). Informes de Gestión I-2015. Florencia: Universidad de la Amazonia.

Sauto, R., Boniolo, P., Dalle, P. y Elbert, R. (2005). Manual de metodología: construcción del marco teórico, formulación de los objetivos y elección de la metodología. Bue- nos Aires: Clacso Libros. Agencia Sueca de Desarrollo Internacional.

Silva L. M. (2011). El primer año universitario: un tramo crítico para el éxito académico. Perfiles educativos, 33(SPE), 102-114. Recuperado de http://www. scielo.org.mx/scielo.php?script $=$ sci_arttext\&pi$\mathrm{d}=$ S0185-26982011000500010\&lng=es\&tlng=es.

United Nations Educational, Scientific and Cultural Organization. [Unesco]. (2004). Cuatro componentes de una educación de calidad para todos los jóvenes. En Una Educación de Calidad para todos los Jóvenes (pp. 83-115). Ginebra: Oficina Internacional de Educación.

Universidad Nacional de Colombia. (2009). Deserción universitaria: un flagelo para la educación superior. $\mathrm{Cla}$ ves para el Debate Público, 27. Bogotá. Recuperado de http://www.alfaguia.org/alfaguiav2/bin/articulos. php?idFicha $=113$ 